\title{
AVALIAÇÃO DA SEGURANÇA DO PACIENTE EM CIRURGIA CARDÍACA DE UM HOSPITAL PÚBLICO
}

\author{
Evaluation of patient safety during cardiac surgery at a public hospital \\ Evaluación de la seguridad del paciente en cirugía cardíaca de un hospital público \\ Marcia Bergamo Giannattasio', Fabio Papa Taniguchi²
}

RESUMO: Objetivo: Avaliar itens de segurança na cirurgia cardíaca em pacientes de um hospital público. Método: Estudo descritivo e transversal. A coleta de dados, desenvolvida em 2012, foi realizada em uma instituição hospitalar pública estadual, onde foram observadas 30 cirurgias cardíacas por meio de um roteiro embasado nas 3 fases do checklist de cirurgia segura da Organização Mundial da Saúde (OMS), com alternativas descritas como "em conformidade" e "em não conformidade". Resultados: Os resultados de não conformidade corresponderam a 56\% dos casos antes da indução anestésica, $57 \%$ antes do procedimento e $75 \%$ na saída da sala de cirurgia. Conclusão: O checklist da OMS se faz necessário, porém, o treinamento profissional e a educação permanente constituem a linha mestra para a formação de uma equipe de saúde crítica e consciente do seu papel na segurança dos pacientes. Palavras-chave: Enfermagem perioperatória. Lista de checagem. Gestão de qualidade.

ABSTRACT: Objective: Evaluation of security items in cardiac surgery at a public hospital. Methods: Cross-sectional descriptive study. Data collection was performed in 2012 at a public state-owned hospital, where 30 cardiac surgeries were observed and evaluated through a script based on the 3 steps of the World Health Organization (WHO) surgical safety checklist. Procedures were categorized as "in conformance" or "in non-conformance." Results: Non-conformance results made up $56 \%$ of cases before anesthetic induction, $57 \%$ prior to the procedure, and $75 \%$ of cases before leaving the operating room. Conclusions: Though the WHO checklist is indispensable, professional training and continuing education remain crucial for training a critical health care team that is aware of its role in securing patient safety.

Keywords: Perioperative nursing. Checklist. Quality Management.

RESUMEN: Objetivo: Evaluar ítems de seguridad en la cirugía cardíaca en pacientes de un hospital público. Método: Estudio descriptivo y transversal. La colecta de datos, desarrollada en 2012, fue realizada en una institución hospitalaria pública estadual, donde fueron observadas 30 cirugías cardíacas por medio de un itinerario basado en las 3 fases del checklist de cirugía segura de la Organización Mundial de la Salud (OMS), con alternativas descritas como "en conformidad" y "en no conformidad". Resultados: Los resultados de no conformidad correspondieron al 56\% de los casos antes de la inducción anestésica, un 57\% antes del procedimiento y un 75\% en la salida del quirófano. Conclusión: El checklist de la OMS se hace necesario, sin embargo, la capacitación profesional y la educación permanente constituyen la línea maestra para la formación de un equipo de salud crítico y consciente de su papel en la seguridad de los pacientes.

Palavras clave: Enfermería perioperatoria. Lista de verificación. Gestión de la calidad.

'Mestranda em Ciências da Saúde do curso de pós Graduação do Instituto de Assistência Médica ao Servidor Público Estadual - IAMSPE. E-mail: sergmarc@uol.com.br Rua dos Donatários, 39, Apto. 52 - Mooca - CEP: 03167-010 - São Paulo (SP), Brasil.

2Doutor em Cirurgia pela Universidade Estadual Paulista “Júlio de Mesquita Filho" - São Paulo (SP), Brasil.

Recebido: 16 maio 2016 - Aprovado: 18 ago. 2016

DOI: $10.5327 / Z 1414-4425201600030002$ 


\section{INTRODUÇÃO}

A publicação To err is human, lançada nos dos Estados Unidos da América (EUA) em 1999, descreve que erros acontecem e são frequentes durante a assistência à saúde, provocando milhares de mortes e sequelas irreversíveis. Nesse país, os erros no sistema de saúde são a oitava causa de morte, apesar dos enormes investimentos nessa área. Essa publicação, apesar de não científica, causou mudanças e promoveu avanços gigantescos na área da pesquisa e medidas práticas para a promoção da segurança do paciente não apenas nos EUA, mas em todo o mundo ${ }^{1}$.

A Organização Mundial da Saúde (OMS) refere que, a cada ano, dezenas de milhões de pacientes no mundo são vítimas de lesões incapacitantes ou mortes por práticas inseguras em saúde ${ }^{2}$. Esses erros afetam 1 em cada 10 pacientes, em média, estimativa ainda mais séria em países em desenvolvimento, onde, de cada 300 pacientes admitidos nos hospitais, 1 morre $^{2}$.

Diante do exposto, a OMS lançou, em outubro de 2004, a Aliança Mundial para a Segurança do Paciente, com o objetivo de descrever desafios para a segurança dos pacientes.

Um dos desafios propostos foi o programa Cirurgias Seguras Salvam Vidas com a utilização de um checklist transoperatório que possibilita uma avaliação integral do paciente, desenvolvido em três fases para o seu seguimento: antes da indução anestésica; antes do início da cirurgia; e antes que o paciente deixe a sala cirúrgica, compreendendo, inclusive, que diferentes cenários de práticas o adaptarão a suas próprias circunstâncias ${ }^{2}$.

Uma pesquisa realizada em 2 hospitais brasileiros verificou que, dos 1.103 pacientes estudados, 56 sofreram eventos adversos. As infecções associadas aos cuidados da saúde e às complicações cirúrgicas e/ ou anestésicas representaram $44,6 \%$ dos danos ${ }^{3}$. Outro estudo, conduzido em 2008 , revelou que 1 em cada 150 pacientes hospitalizados morre em decorrência de um incidente, e que quase dois terços dos eventos adversos foram relacionados à assistência cirúrgica ${ }^{4}$.

Diversas razões ainda são apontadas para a ocorrência de efeitos adversos na cirurgia, como a precária comunicação e a distração entre a equipe multiprofissional, a não conferência da identificação do paciente e de materiais durante a assistência, entre outros, que constituem indicadores importantes para elevar a propensão de erros ${ }^{4}$.

Ressalta-se que listas de verificação cirúrgicas têm sido eficazes em minimizar os eventos adversos, favorecendo a segurança dos pacientes.
Nesse contexto, a utilização do checklist nas instituições hospitalares brasileiras vem crescendo, com investimento no trabalho em equipe e desenvolvimento de protocolos e indicadores pertinentes ao assunto. Órgãos públicos e profissionais do Brasil desenvolvem estratégias de auxílio às instituições, contemplando nosso maior avanço na primeira Portaria, $\mathrm{n}^{\circ}$ 529/2013, do Ministério da Saúde, com foco no Programa Nacional de Segurança do Paciente, instrumento que prevê diversos protocolos, normas e guias a serem aplicados pelas instituições hospitalares com o objetivo de garantir a segurança do paciente 5 .

Acreditamos que, embora exista a preocupação das instituições com a segurança dos pacientes, é preciso persistir em uma prática evidenciada na assistência cirúrgica. A partir dessa afirmativa, surgiu o seguinte questionamento: um procedimento cirúrgico é seguro sem a utilização do checklist?

Tendo em vista que a segurança deve estar presente em todos os procedimentos cirúrgicos, especificamos um estudo sobre os procedimentos anestésicos cirúrgicos cardíacos, por serem de alta complexidade e de grande valorização no Brasil. Publicada pela Sociedade Brasileira de Cirurgias Cardiovasculares (SBCCV), a pesquisa aponta o Brasil como o segundo país do mundo em número de cirurgias cardíacas realizadas anualmente, totalizando cerca de 102 mil cirurgias/ano, atrás apenas dos EUA' .

\section{OBJETIVO}

Avaliar a conformidade ao checklist referente aos itens de segurança na cirurgia cardíaca em pacientes de um hospital público.

\section{MÉTODO}

Trata-se de um estudo observacional e transversal. Após aprovação pelo Comitê de Ética em Pesquisa da instituição envolvida, a pesquisa foi realizada, em março de 2012, no Centro Cirúrgico do Hospital do Servidor Público Estadual de São Paulo, cadastrado no Sistema Nacional de Informação sobre Ética em Pesquisa (SISNEP), sob protocolo FR-441480.

O instrumento foi elaborado com 30 variáveis, nomeado como instrumento de formulário de observação sistematizada adaptado ao checklist da OMS. Como critérios de inclusão foram escolhidos os procedimentos eletivos de cirurgias cardíacas por toracotomia médio-esternal, com circulação 
extracorpórea (CEC) em pacientes de ambos os sexos, acima de 21 anos. O pesquisador observou, na íntegra, 30 procedimentos, uma amostra significativa que representou $68,18 \%$ dos procedimentos cirúrgicos cardíacos realizados nessa instituição no período escolhido para a coleta de dados.

Os itens de observação (variáveis) foram divididos em etapas: na $1^{\text {a }}$ etapa foi feita a identificação do observado; na $2^{\circ}$ etapa foram observados 19 itens antes da indução anestésica; na $3^{\text {a }}$ etapa, 7 itens foram observados antes do início do procedimento cirúrgico; na $4^{\mathrm{a}}$ etapa, quatro itens observados antes da saída do paciente da sala. O registro desses itens foi descrito por meio de categorização nominal binária, sendo que cada item tinha como opção de resposta "sim" (em conformidade) ou "não" (não conformidade).

Os dados são apresentados em números absolutos e percentuais, e foram submetidos à análise estatística descritiva com o auxílio do programa Microsoft Excel ${ }^{\mathbb{B}}$.

\section{RESULTADOS}

$\mathrm{Na} 1^{\mathrm{a}}$ etapa, dos 30 procedimentos observados, $70 \%(\mathrm{n}=21)$ dos pacientes eram do sexo masculino, $43 \%(n=13)$ na faixa etária de 61 a 70 anos. Em relação ao procedimento realizado, observou-se que $90 \%(\mathrm{n}=27)$ realizaram a revascularização do miocárdio.

$\mathrm{Na} 2^{\mathrm{a}}$ etapa, antes da indução anestésica, dos 19 itens observados, detectou-se que $8(44 \%)$ apresentam-se "em conformidade", e 11 (56\%), "em não conformidade". Em relação aos resultados da terceira etapa, dos 7 itens observados, foram detectados 4 (57\%) "em conformidade" e 3 (43\%) "em não conformidade". Como resultados da quarta e última etapa, obtivemos 1 (25\%) item "em conformidade" e 3 (75\%) "em não conformidade" (Quadro 1).

Dos 30 itens observados, 17 (57\%) foram elencados "em não conformidade".

\section{DISCUSSÃO}

\section{Primeira etapa: dados de identificação}

O sexo masculino é predominante em relação ao feminino. Em estudo realizado na Região Sul do Brasil, o perfil epidemiológico dos pacientes submetidos à cirurgia cardíaca demonstra que a maioria dos procedimentos é composta pela revascularização do miocárdio e em pacientes com idade avançada (acima de 70 anos), resultados compatíveis com este estudo?.

\section{Segunda etapa: antes da indução anestésica}

Os dados demonstram que a identificação dos pacientes não é realizada, apesar de estar prevista na Lei ${ }^{\circ} 10.241$, de 17 de março de 1999, que dispõe sobre os direitos dos pacientes, usuários dos serviços de saúde do Estado de São Paulo e prevê que o paciente tem o direito de ser identificado8.

A Joint Comission International, entidade norte-americana que certifica serviços de saúde, publicou um desafio nacional americano para segurança dos pacientes, alertando a respeito da importância e da necessidade da inclusão da pulseira de identificação. Em vista da ocorrência de erros relacionados à inadequada identificação do paciente, esse desafio permanece como meta para as instituições de saúde que buscam a certificação de qualidade 9 .

Destacamos, em nossa observação, que a equipe médica cirúrgica conhece o paciente, porém em momento algum há conferência do paciente com toda a equipe da sala operatória.

É frequente a realização de estudos que objetivam qualificar a comunicação entre as unidades e a conferência rigorosa dos dados de identificação dos pacientes ${ }^{3,4}$.Indagamos sobre a falta do consentimento informado cirúrgico e anestésico do paciente, visto que tal prática é realizada em outros setores da instituição. É evidente que o tratamento cirúrgico deve ser feito com foco no paciente como indivíduo, com apresentação e análise de riscos e benefícios centrados naquele paciente específico, esclarecimento de direito do cidadão e responsabilidade do profissional médico ${ }^{8}$. Verificamos que essa prática existe por parte dos cirurgiões e anestesistas, porém sem o consentimento informado e assinado pelo paciente.

Em relação ao conhecimento sobre as alergias do paciente, a não conformidade encontrada nos faz pensar quanto à possibilidade dos eventos adversos ocorrerem com mais frequência.

Nos EUA, pelo menos 1,5 milhão de pessoas são prejudicadas anualmente pela administração errada de drogas. Estudo verificou que erros perioperatórios contribuem significativamente para essa estatística e, inclusive, apontou diversos motivos, dentre eles a falta de conhecimento sobre as alergias dos pacientes ${ }^{2}$.

O projeto Closed Claims (acionamentos fechados), da Sociedade Americana de Anestesiologistas, notou que os erros na administração de drogas resultaram em sérios problemas, incluindo a morte em $24 \%$ e morbidade importante em $34 \%$ dos casos revisados ${ }^{10}$. 
Embora seja difícil fornecer uma estimativa total precisa da extensão dos danos atribuíveis aos erros de medicação perioperatórias, é quase certo que erros nocivos são subnotificados. Refletindo sobre essa problemática e considerando adequada a checagem de possíveis alergias dos pacientes, entendemos que uma intercorrência indesejável relacionada com alergias é prevenível se houver conferência sistematizada e comunicação efetiva entre os membros da equipe de cuidado.

Quadro 1. Resultados do instrumento de formulário de observação sistematizada adaptado ao checklist da Organização Mundial de Saúde, São Paulo (SP), 2012.

\begin{tabular}{|c|c|c|c|c|c|}
\hline \multirow{2}{*}{\multicolumn{2}{|c|}{ Itens observados }} & Sim & $\%$ & Não & $\%$ \\
\hline & & \multicolumn{2}{|c|}{ Conformidade } & \multicolumn{2}{|c|}{ Não conformidade } \\
\hline \multicolumn{6}{|c|}{ Antes da indução anestésica } \\
\hline 1 & Identificação do paciente - pulseira & 3 & 10 & 27 & 90 \\
\hline 2 & Prontuário conferido & - & 0 & 30 & 100 \\
\hline 3 & Consentimento informado & - & 0 & 30 & 100 \\
\hline 4 & Paciente possui alergia conhecida & - & 0 & 30 & 100 \\
\hline 5 & Durante a anestesia a equipe cirúrgica em sala & - & 0 & 30 & 100 \\
\hline 6 & Durante a anestesia a equipe de enfermagem em sala & 30 & 100 & - & 0 \\
\hline 7 & Monitorização & 30 & 100 & - & 0 \\
\hline 8 & Conferência dos equipamentos anestésicos & 30 & 100 & - & 0 \\
\hline 9 & Foi detectada via aérea difícil & - & 0 & 30 & 100 \\
\hline 10 & Existe um material especifico para vias aéreas difíceis & - & 0 & 30 & 100 \\
\hline 11 & Profilaxia antimicrobiana realizada 30 a 60 minutos antes da incisão & - & 0 & 30 & 100 \\
\hline 12 & Instrumentais com indicadores químicos & - & 0 & 30 & 100 \\
\hline 13 & Equipamentos em sala com seus acessórios & 30 & 100 & - & 0 \\
\hline 14 & Consignados em sala conferidos & 30 & 100 & - & 0 \\
\hline 15 & CEC em sala com acessórios & 30 & 100 & - & 0 \\
\hline 16 & Reposição sangue em sala & - & 0 & 30 & 100 \\
\hline 17 & Preparado material para acessos venosos e arteriais & 30 & 100 & - & 0 \\
\hline 18 & Preparado material de sondagem vesical & 30 & 100 & - & 0 \\
\hline 19 & Exames de imagem em sala & - & 0 & 30 & 100 \\
\hline \multicolumn{6}{|c|}{ Antes da incisão cirúrgica } \\
\hline 20 & Mantida pulseira de identificação & - & 0 & 30 & 100 \\
\hline 21 & Equipe cirúrgica completa em sala & - & 0 & 30 & 100 \\
\hline 22 & Equipe de enfermagem em sala & 30 & 100 & - & 0 \\
\hline 23 & Paciente monitorizado & 30 & 100 & - & 0 \\
\hline 24 & Desfibrilador ligado com pás internas & 30 & 100 & - & 0 \\
\hline 25 & A CEC está montada & 30 & 100 & - & 0 \\
\hline 26 & Houve em algum momento eventos críticos previstos & - & 0 & 30 & 100 \\
\hline \multicolumn{6}{|c|}{ Antes da saída do paciente da sala } \\
\hline 27 & Registros realizados & 30 & 100 & - & 0 \\
\hline 28 & Contagem de instrumentos e gazes & - & 0 & 30 & 100 \\
\hline 29 & Conferência de pulseira de identificação & - & 0 & 30 & 100 \\
\hline 30 & Transferência com segurança & - & 0 & 30 & 100 \\
\hline
\end{tabular}

CEC: circulação extracorpórea. 
Ressaltamos novamente as falhas de comunicação quando observamos a ausência da equipe cirúrgica completa em sala, pois esse cenário indica que a comunicação entre os profissionais está afetada. Embora a equipe de enfermagem estivesse presente, ausentou-se várias vezes para buscar material, o que representa uma montagem de sala inadequada. Todos os envolvidos no procedimento anestésico-cirúrgico devem permanecer em sala constantemente, evitando-se falhas de comunicação, consideradas um fator de insegurança².

Outra preocupação dos autores é em relação aos procedimentos anestésicos, visto que não há uma conferência ou checagem de equipamentos sistematizada; a verificação existente é feita individualmente pelo anestesista e conforme seu entendimento, podendo gerar intercorrências inesperadas, porém, evitáveis.

A provisão segura de anestesiologia depende de uma preparação cuidadosa envolvendo não só uma conferência sistematizada do anestesista momentos antes do procedimento anestésico, mas também de uma manutenção preventiva, diária e permanente de todos os equipamentos que envolvem a anestesia por outros profissionais conhecedores do equipamento ${ }^{11}$.

Consideramos bastante expressivos os riscos inerentes à infecção cirúrgica, pois dados observados demonstraram a profilaxia antimicrobiana inadequada, o preparo de acessos sanguíneos e a sondagem vesical com técnicas assépticas inseguras e, ainda, a falta de indicadores químicos nos instrumentais utilizados. Inferimos que um procedimento cirúrgico cardíaco, por si só, já é considerado vulnerável para infecções, devido à sua alta complexidade; assim, a exposição às infecções se torna ainda mais provável com a prática encontrada.

Estudo descreve que a profilaxia antimicrobiana é indicada quando existe risco elevado para infecção, como em pacientes idosos, diabéticos e imunodeprimidos, além de cirurgias de alta complexidade e com utilização de prótese. O uso inadequado desse recurso pode favorecer a seleção de cepas bacterianas resistentes. Especialistas americanos chegaram ao consenso de que, em geral, a droga seja iniciada entre 30 e 60 minutos antes da incisão ${ }^{12}$.

Contudo, o emprego de antibiótico durante a profilaxia não é a mais importante medida para prevenir infecções cirúrgicas; a identificação de riscos, o adequado preparo pré -operatório e o emprego de técnicas cirúrgicas assépticas são práticas primordiais para um bom resultado. Além disso, deve-se dar atenção à limpeza do ambiente, ao uso de uniforme cirúrgico e à esterilização adequada dos instrumentais cirúrgicos ${ }^{12,13}$.

Uma pesquisa revelou que em $83 \%$ dos casos estudados houve comprometimento da segurança em relação à exposição e à transmissão de microrganismos durante o ato anestésico cirúrgico, em decorrência de comportamentos negativos de toda a equipe da sala operatória ${ }^{13}$.

Houve conformidade nas categorias de equipamentos, seus acessórios e consignados conferidos. Essa questão também é citada como uma estratégia importante para se evitar estresse transoperatório, e embora na instituição estudada não exista uma conferência sistematizada, há uma preocupação somente do circulante de sala, sem a checagem em equipe.

Quanto à CEC, à conferência e ao controle do equipamento, são feitos de acordo, porém, sempre de forma individual pelo profissional responsável, o perfusionista.

Nos procedimentos cardíacos é rotina haver reposição sanguínea em sala operatória, porém, tal prática não foi observada no local pesquisado. Em estudo específico sobre os riscos aos pacientes de cirurgia de alta complexidade no período intraoperatório, a questão do desequilíbrio no volume de líquidos é apontada ${ }^{13}$.

$\mathrm{Na}$ análise dessa etapa apontamos como mais relevantes os itens da comunicação ineficaz entre os membros da equipe, a identificação dos pacientes ausentes e os riscos de infecções.

\section{Terceira etapa: antes da incisão cirúrgica}

Realçamos nessa etapa a constante ausência da identificação do paciente e a equipe cirúrgica incompleta, fatores que configuraram uma cirurgia insegura; contudo, os procedimentos cirúrgicos foram iniciados com a perfeita monitorização do paciente com os equipamentos e acessórios preparados especificamente para a cirurgia específica, o que nos trouxe certo conforto.

A OMS sugere que nessa fase haja uma comunicação efetiva da equipe em relação aos possíveis eventos previstos, o que não foi observado ${ }^{2}$.

As equipes cirúrgicas parecem reconhecer que as falhas na comunicação podem ser uma barreira fundamental para a assistência segura e efetiva, mas, mesmo assim, são encontradas falhas ${ }^{4,13}$.

Na interpretação dos autores, nessa fase, o maior destaque está nas falhas de comunicação entre os membros da equipe. 


\section{Quarta etapa: antes do paciente sair da sala cirúrgica}

Nessa etapa observamos que não há uma rotina para contagem de artigos cirúrgicos ao final do procedimento. O Manual da OMS descreve a importância do rastreamento geral de itens na sala operatória, em que cada serviço deve ter uma norma especificando quando e por quem devem ser realizadas as contagens cirúrgicas, quais itens devem ser contados e como as contagens devem ser documentadas ${ }^{2}$.

O Conselho Regional de Medicina do Estado de São Paulo descreve que, ao final do ato operatório, a contagem sistemática das compressas é obrigatória e deve ser verificada e confirmada pelo cirurgião. Não pode haver dúvidas e, se houver, deve-se solicitar a realização radiografias simples do sítio cirúrgico ${ }^{14}$.

Um estudo brasileiro de autoria médica descreve que a cirurgia representa um ato profissional no qual participaram vários integrantes (membros das equipes cirúrgica, anestesista e de enfermagem de sala). Questiona-se até que ponto em um ato operatório poderia ser o cirurgião responsável pelas falhas dos demais membros da equipe, visto que cada componente tem uma nítida divisão de tarefas ${ }^{15}$. Nesse contexto, reforçamos a importância do uso do checklist, atividade realizada em equipe, na qual todos os membros são responsáveis pela cirurgia segura.

Evidenciamos, nessa etapa, a contínua falta de identificação do paciente, situação que na transferência de unidade se torna ainda mais grave e insegura, pois o paciente está totalmente vulnerável, podendo ser submetido a procedimentos indevidos.

O transporte do paciente foi realizado por parte da equipe cirúrgica acompanhada do circulante de enfermagem, mas verificou-se a ausência do enfermeiro em todos os casos observados.

Em estudo descreve-se que durante o encaminhamento do paciente da sala operatória para a unidade de terapia intensiva ou semi-intensiva, o ideal é que o enfermeiro acompanhe o processo de transporte com a equipe cirúrgica anestésica e passe o plantão ao enfermeiro daquela unidade ${ }^{16}$, fatos ausentes em nossa observação. Durante nossa observação, houve a preocupação, por parte da equipe, de transportar o paciente com segurança, porém a falta de equipamentos em condições favoráveis para essa prática tornou morosa a transferência, causando atrasos e riscos para o paciente.

Os autores compreenderam que nessa última etapa a identificação ausente dos pacientes é o item de maior preocupação.

\section{CONCLUSÃO}

A instituição de saúde deseja melhorar a segurança de seus pacientes; assim, sugerimos que antes de qualquer ação é preciso investir em uma política interna de segurança do paciente como primeira e principal diretriz do planejamento institucional. É preciso expor os objetivos do programa Cirurgias Seguras Salvam Vidas e a sua importância para todos os profissionais de saúde, tornando esse contexto um compromisso coletivo.

Conhecer as falhas de segurança cirúrgica de um centro cirúrgico específico nos faz pensar que é evidente a necessidade da aplicação do checklist cirúrgico nessa instituição, porém, o desafio é muito maior do que a aplicação de um impresso, é preciso um olhar direcionado para a segurança do paciente em toda a instituição, para que o checklist não seja mais um impresso a ser preenchido, mas sim um instrumento de segurança efetivo.

Com este estudo, entendemos a necessidade de uma ação imediata em relação ao investimento na identificação contínua dos pacientes e à valorização da comunicação efetiva da equipe, pois os achados demonstraram-se desfavoráveis e relevantes. É necessário, ainda, compreender que o predomínio de práticas inseguras em relação à infecção cirúrgica potencializa os riscos operatórios, portanto, sugerimos o ajuste imediato com o auxílio de uma educação permanente com todos profissionais envolvidos em um procedimento cirúrgico.

Concluímos que o checklist é necessário para que as cirurgias cardíacas se tornem seguras na instituição, visto que esta, embora tenha um envolvimento positivo dos profissionais no transoperatório, apresenta falhas que podem ser minimizadas com o uso consciencioso do checklist. 


\section{REFERÊNCIAS}

1. Kohn LT, Corrigan JM, Donaldson MS, McKay T, Pike KC. To err is human. Washington: National Academy Press, 2000.

2. World Health Organization. Safe surgery saves lives. The second global patient safety challenge. Geneva: World Health Organization, 2009.

3. Boeckmann LMM, Rodrigues MCS. Segurança cirúrgica na cesárea: revisão integrativa. Cogitare Enferm. 2015;20(4):758-76.

4. Bohomol E, Tartali JAA. Adverse effects in surgical patients: knowledge of the nursing professional. Acta Paul Enferm. 2013;26(4):376-81 [acesso em 2016 jul. 21]. Disponível em: http://www.scielo.br/scielo. php?script=sci_arttext\&pid=S0103-21002013000400012\&lng=en

5. Brasil. Ministério da Saúde. Portaria n 529, de 1º de abril de 2013. Institui o Programa Nacional de Segurança do Paciente (PNSP). Brasília: Ministério da Saúde, 2013 [acesso em 2015 mar. 04]. Disponível em: http://bvsms.saude.gov.br/bvs/saudelegis/gm/2013/ prt0529_01_04_2013.html

6. Gomes W. Brasil é o segundo país do mundo em cirurgias cardíacas, 2013 [acesso em 2015 jul. 25]. Disponível em: http://saudeweb.com. $\mathrm{br} / 30501 /$ brasil-e-o-segundo-pais-do-mundo-em-cirurgias-cardiacas/

7. Bonotto JM, Boer LA. Estudo sobre o perfil do paciente submetido à cirurgia de revascularização do miocárdio, em um hospital da região sul catarinense, 2013 [acesso em 2015 jan. 27]. Disponível em: http://www.uniedu.sed.sc.gov.br/wp-content/uploads/2013/10/ Janete-Morangoni-Bonotto.pdf

8. Brasil. Ministério da Saúde. LEGIS - Sistema de Legislação da saúde. Dispõe sobre os direitos dos usuários dos serviços e das ações de saúde no Estado e dá outras providências. Brasília: Ministério da Saúde, 2009 [acesso em 2014 ago. 15]. Disponível em: http:// governo-sp.jusbrasil.com.br/legislacao/168477/lei-10241-99
9. The Joint Commission. Universal protocol, 2013 [acesso em 2016 jul. 16]. Disponível em: http://www.jointcommission.org/PatientSafety/ UniversalProtocol/

10. Bowdle TA. Drug administration errors from the ASA closed claims project. ASA Newsletter. 2003;67(6):11-3.

11. Paranaguá TTB, Bezerra ALQ, Silva AEBC, Azevedo Filho FM. Prevalence of no harm incidents and adverse events in a surgical clinic. Acta Paul Enferm. 2013;26(3):256-62. [acesso em 2016 jul. 27]. Disponível em: http://www.scielo.br/ scielo.php?script=sci_arttext\&pid=S0103-21002013000300009 $\& \operatorname{lng}=\mathrm{pt}$

12. Barreto RASS, Barbosa MA, Souza ACS. A segurança do paciente na sala operatória: incidentes críticos relacionados à prevenção de infecção de sítio cirúrgico, 2010 [acesso em 2015 fev. 02]. Disponível em: http://www.sbpcnet.org.br/livro/63ra/conpeex/doutorado/ trabalhos-doutorado/doutorado-regiane-aparecida.pdf

13. Pancieri AP, Santos BP, Avila MAG, Braga EM. Checklist de cirurgia segura: análise da segurança e comunicação das equipes de um hospital escola. Rev Gaúcha Enferm. 2013;34(1):71-8.

14. Conselho Regional de Medicina de São Paulo. Corpos estranhos intra-abdominais. Jornal do Cremesp, 2005 [acesso em 2016 jul. 20]. Disponível em: http://www.cremesp.org.br/?siteAcao= Jornal\&id $=590$

15. Filho GR, Silva LFN, Ferracini AM, Bahr GL. Protocolo de Cirurgia Segura da OMS: o grau de conhecimento dos ortopedistas brasileiros. Rev Bras Ortop. 2013;48(6):554-62.

16. Meeker MH, Rothrock JC. Alexander Cuidados de enfermagem ao paciente cirúrgico. 10a ed. Rio de Janeiro: Guanabara Koogan; 1997. 